\title{
REFLEXIONES SOBRE LOS TIPOS DE INTERVENCIÓN DE CORVALÁN. A PROPÓSITO DEL CASO DE BUENAVENTURA
}

\section{RESUMEN}

\author{
Diani Rocío Mesa López* \\ Maura Elizabeth Vera Pérez** iD
}

El abordaje sociológico de la intervención social implica, entre otras cosas, el estudio de los procesos de formulación y ejecución de iniciativas que buscan la transformación social. Dichos procesos no se presentan de forma homogénea, sino que se configuran en diferentes tipos de intervención definidos por diversas visiones de cambio, tal como lo plantea Corvalán. Por tal motivo, en el presente artículo se propone comprender estas particularidades de la intervención tomando el caso concreto del municipio de Buenaventura, en el periodo 2000-2017. Para esto, se realiza un estudio de tipo cualitativo desarrollado a través de una revisión documental. Se concluye que, para el caso de Buenaventura, la intervención social se ha desarrollado a partir de varias perspectivas que se entrecruzan y persisten en el tiempo.

Palabras clave: Buenaventura, Corvalán, gestión asociada, tipos de intervención.

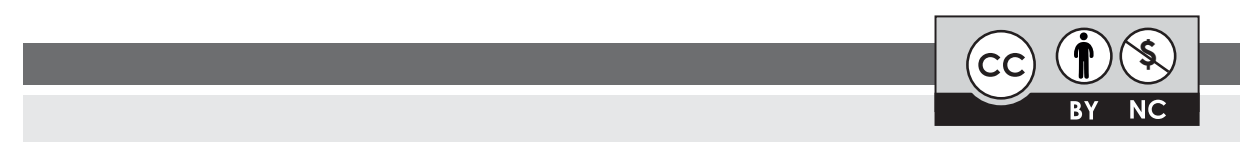

Recibido: 14 de noviembre de 2019 | Aprobado: 16 de febrero de 2020.

\section{CÓMO CITAR ESTE ARTÍCULO:}

Mesa López, D. R., \& Vera Pérez, M. E. (2020). Reflexiones sobre los tipos de intervención de Corvalán. A propósito del caso de Buenaventura. Collectivus, Revista de Ciencias Sociales, 7 (2), 145-162. DOI: https://doi.org/10.15648/Collectivus.vol7num2.2020.2678

\footnotetext{
* Socióloga de la Universidad del Valle. Colombia, Valle del Cauca, Cali Cr 4B \#44-21. diani.mesa@correounivalle.edu.co

** Socióloga de la Universidad del Valle. Colombia, Valle del Cauca, Cali Cr 1FN \# 76-46. maura.vera@correounivalle.edu.co
} 


\title{
REFLECTIONS ON THE TYPES OF INTERVENTION OF CORVALÁN. ON THE PURPOSE OF THE CASE OF BUENAVENTURA
}

\begin{abstract}
The sociological approach to social intervention implies, among other things, the study of the processes of formulation and execution of initiatives that seek social transformation. These processes are not presented in a homogeneous way, but are configured in different types of intervention defined by different visions of change, as stated by Corvalán. For this reason, this article proposes to understand these particularities of the intervention taking the specific case of the municipality of Buenaventura, in the period 2000-2017. For this, a qualitative study developed through a documentary review is carried out. It is concluded that, in the case of Buenaventura, social intervention has developed from several perspectives that intersect and persist over time.
\end{abstract}

Keywords: associated management, Buenaventura, Corvalán, social intervention.

\section{Introducción}

Para entrar en materia, es importante explicar que Buenaventura es reconocido como uno de los municipios que más recursos naturales ostenta en Colombia. Además, conserva una privilegiada ubicación geoestratégica que convierte a su puerto en el más importante a nivel nacional y en uno de los diez principales de América Latina, y moviliza más del $70 \%$ de la mercancía que entra y sale del país (Contralora Distrital de Buenaventura, 2017). Atributos que le valieron su nombramiento como Distrito Especial, Industrial, Portuario, Biodiverso y Ecoturístico en el año 2007.

Ahora bien, pese a la extraordinaria riqueza de la oferta natural y su importancia en el sector comercial, en Buenaventura habita una de las poblaciones con menor calidad de vida en Colombia, pues se encuentra entre los municipios más pobres de los cuarenta y siete que conforman el litoral Pacífico, con un $41 \%$ de personas bajo esta condición, mientras que el promedio nacional es del $20 \%$ (DANE, 2018).

Bajo este panorama, Buenaventura se ha convertido en objeto de numerosas intervenciones que buscan dar respuesta a dichas problemáticas a través de políticas, planes, programas, proyectos e iniciativas locales (o comunitarias). Estas han sido desarrolladas desde una amplitud de áreas que abarcan lo social, económico, cultural y ambiental. Y que han sido llevadas a cabo por diversos actores, tales como el Estado, empresas privadas, ONG y organizaciones comunitarias.

Cada uno de los actores de los que intervienen en Buenaventura, lo hacen desde sus propias perspectivas de lo que se debe cambiar. Así, para algunos, la solución está en la generación de ingresos a partir de la puesta en marcha de proyectos productivos, y por esta misma línea, en el fortalecimiento empresarial a través de grandes inyecciones de capital en el sector portuario; otros, en cambio, enfatizan en educación, vivienda digna, orden público, entre otros. Igualmente, están aquellas iniciativas que rompen con esta dicotomía y desarrollan procesos basados en modelos híbridos que combinan varias perspectivas. Tal como el «Plan Maestro 2050: la Buenaventura que nos merecemos». A partir de este ambicioso plan, se buscó intervenir en el municipio a partir de cinco ejes:

*Buenaventura social, cultural y deportivo

*Buenaventura activa: promoción económica 
*Buenaventura territorial: urbanismo, infraestructura y medio ambiente

*Buenaventura logística y portuaria: competitividad e integración regional

*Buenaventura institucional: gobernanza.

Su importancia radica en que, además de involucrar a distintos actores e intervenir en varios ejes, de él se desprenden algunos de los proyectos que fueron contemplados en la presente investigación.

Hay varios autores dentro del campo de las ciencias sociales, que han reflexionado acerca de los distintos tipos de intervención, tanto desde la sociología como desde el trabajo social. No obstante, para la presente investigación se retoman autores como Corvalán (1996), y Carballeda [2002] (2005), quienes brindan los planteamientos teóricos, a partir de los cuales se logra construir una tipología de las intervenciones, realizadas en Buenaventura durante el periodo 2000-2017. En este proceso se identifican cuatro tipos que se han desarrollado a lo largo de estos años: intervención integradora, competitiva, movilizadora con rasgos de tipo militantista y, finalmente, intervención mixta o de gestión asociada (híbrida).

A continuación, se presenta el estado del arte, la metodología de investigación, seguida de las perspectivas teóricas alrededor de la intervención social. En los siguientes apartados se plasman los resultados y su discusión. Por último, se exponen las conclusiones, que plantean cuáles han sido las perspectivas de cambio predominantes en Buenaventura.

\section{Estado de la cuestión}

Durante mucho tiempo, Buenaventura fue vista principalmente desde su pertenencia a la región Pacífica. Esto se explica porque dicho municipio, junto a los demás que conforman esta zona del país, comparten innumerables características; entre ellas su población mayormente afrocolombiana, condiciones socioeconómicas, rica biodiversidad y clima tropical. De ahí que gran parte de los estudios que se refieren a Buenaventura, tienen precisamente este enfoque regional. Entre los autores que han realizado dichos estudios se encuentran: Oslender [1999] (2012), Escobar (2004), y Castillo (2006), quienes exponen aspectos sobre las dinámicas sociales y económicas que rodean la intervención social en el Pacífico, concretamente, la extracción y explotación de recursos naturales, y el aprovechamiento de su posición geográfica para la ejecución de actividades económicas.

Tal como afirma Escobar (2004), las distintas intervenciones realizadas en la región Pacífica, han tenido el propósito de alcanzar el desarrollo característico de los países del primer mundo, empeorando la condición de pobreza y marginación de la población. El autor se refiere a esto como "descontextualización” (noción inspirada en Giddens), que ocurre cuando la modernidad arranca la vida local de su contexto, pues es producida por lo translocal. Así, el Estado ha materializado proyectos espaciales, conquistas de territorios y pueblos; provocando no solo la explotación de recursos naturales, sino también la destrucción de las tradiciones y la cultura de los pueblos étnicos que hacen parte de la región Pacífica. Esto se convierte en uno de los principales obstáculos, por el que no se ha logrado un mayor desarrollo del litoral Pacífico, quedándose en su construcción como entidad desarrollable (Motta, 1995, citada en Escobar, 2004). 
Por lo que se refiere a las poblaciones del Pacífico que fueron afectadas por este modelo de desarrollo capitalista adoptado por el Estado, vemos que movimientos sociales y organizaciones afrocolombianas ejecutaron acciones de resistencia ante la destrucción de sus recursos naturales y degradación de sus tradiciones (Oslender, [1999] 2012; Castillo 2006; Espinosa, 2011).

De ahí que, tal como lo señala Castillo, surgiera el moderno movimiento de negritudes, que buscaba el reconocimiento de sus derechos como grupo étnico anclado a un territorio ancestral con una fuerte identificación con la tierra -o la naturaleza- Este movimiento, en su comienzo se conformó por intelectuales y varios sectores de la población afro, que poco a poco tomaron conciencia de la desatención histórica por parte del Estado, la marginalidad y discriminación a la que eran sometidos por la nación mestiza. De acuerdo con este autor, dichos sectores se oponen al impacto que tienen las intervenciones privadas y del Estado, al territorio del cual son parte. Lo hacen mediante la construcción de discursos, que manifiestan los efectos inmediatos del "modelo de desarrollo que se aplica en la Costa Pacífica, [que] será la destrucción de la selva, el bosque y sistema de ríos" (p.313).

Así también, Oslender [1999] (2012), reflexiona sobre la conformación de los movimientos sociales de las negritudes, como una expresión de la lucha permanente entre lo global y lo local. Es decir, la forma en la cual el Pacífico colombiano es objeto de la expansión de procesos globales, que instauran un capitalismo desarrollista con inversiones privadas y estatales para su aprovechamiento con fines económicos. Pero al mismo tiempo, desde lo local se configuraron proyectos organizativos de resistencia que ubican a la región Pacífica, como un espacio de representación rico en simbolismo y significado cultural. Este proceso de resistencia se ve reflejado en las negociaciones intensas del Estado y las comunidades negras en el reconocimiento de "derechos etnoculturales, o estilos de vida diferentes a las tierras que las comunidades negras han ocupado desde hace varios siglos" (p.210).

Una de las organizaciones más representativas de estos procesos, sea el Proceso de Comunidades Negras, PCN, que estudia Espinosa (2011), y define como un actor red. Es decir, como aquel que establece alianzas, movilizaciones y asociaciones con otros actores y garantiza así el logro de sus metas (p.24). Así mismo, sostiene que este se encuentra inmerso en la dinámica de un conflicto armado, que es apoyado por los modelos de intervención capitalista, los cuales están dirigidos al aprovechamiento de los recursos naturales, por medio del desalojo y expropiación de las comunidades afro de sus territorios. Por lo cual, la autora habla de cómo en este contexto de un interés económico sobre la región, se pone en juego la supervivencia de los grupos étnicos, que se han tenido que "organizar de manera conjunta para defender el derecho a la tierra y luchar contra la degradación ambiental del territorio" (p.37), mediante alianzas tanto locales y globales.

\section{Metodología}

Teniendo en cuenta el objetivo de la investigación, la ruta metodológica estuvo centrada en la revisión de documentos. Dichas fuentes se constituyeron en una herramienta principal para obtener información sobre las políticas, planes, programas y proyectos llevados a cabo en Buenaventura durante el periodo 2000-2017, así como el contexto en el que estas intervenciones se desarrollaron. Ahora bien, a partir de los planteamientos de Tancara (1993), se logra tener una idea clara del significado e importancia de la investigación documental: 
Una serie de métodos y técnicas de búsqueda, procesamiento y almacenamiento de la información contenida en los documentos, en primera instancia, y la presentación sistemática, coherente y suficientemente argumentada de nueva información en un documento científico, en segunda instancia. De este modo, no debe entenderse ni agotarse la investigación documental como la simple búsqueda de documentos relativos a un tema (párr. 4).

Las fuentes documentales, permiten realizar un ejercicio retrospectivo de los acontecimientos, por lo cual son una herramienta de gran valor para la presente investigación. No obstante, al igual que otras fuentes, estas también tienen sus desventajas. Si bien los documentos consultados, permitieron acceder a un gran número de intervenciones desarrolladas por el Estado, agentes privados, Tercer sector y organizaciones comunitarias, en muchos casos no profundizaban en aspectos clave como desarrollo y resultados de estos procesos. No obstante, brindaron información fundamental que permitió hacer un balance de las distintas intervenciones y clasificarlas a partir de la construcción de una tipología.

Se consultaron documentos escritos, fotografías y videos. Principalmente en formato digital. Desde luego, las fuentes que proporcionaron dicha información se evaluaron bajo los criterios de autenticidad, credibilidad, representatividad y significado (Scott, 1990 y Scott, 2006). Estas son los siguientes:

- Publicaciones e informes de instituciones estatales: Conpes dirigidos a la región Pacífica y Buenaventura; planes de desarrollo territorial a nivel departamental y distrital. Además, documentos del Ministerio de Cultura y entidades como Findeter, Fedesarrollo entre otros. Los segundos son: informes de la Defensoría del Pueblo, el Centro Nacional de Memoria Histórica y entidades públicas que abordan a nivel regional y local la problemática del municipio. Estos documentos, sirvieron principalmente para identificar la forma en la que se ha planificado y ejecutado la intervención en Buenaventura bajo las instituciones gubernamentales.

- Material visual y publicaciones en redes sociales: Fotografías, infografías, videos y folletos, accediendo a ellos a través de redes sociales (Facebook, Twitter, YouTube) y blogs. Estos brindaron información de las organizaciones de Buenaventura y sus acciones.

- Recopilación de prensa (El País, El tiempo, El Espectador, Semana, 2orrillas, la silla vacía) con noticias de Buenaventura para el periodo 2000-2017, que colaboraron en la construcción de la cronología, y proporcionaron información valiosa sobre el contexto en sus tres niveles: internacional, nacional y local.

- Producción académica: se consultaron tesis doctorales, de maestría, artículos de revista, capítulos de libros, entre otros. Que brindaron una mirada analítica de cómo se ha abordado el estudio de la intervención en Buenaventura, cuáles han sido los enfoques y las perspectivas.

La información proporcionada por estas fuentes, fue sintetizada en una base de datos en Excel construida para el proyecto ${ }^{1}$ más amplio y fichas de lectura. Se recopilaron un total de ciento quince proyectos dirigidos a Buenaventura dentro del periodo estudiado. Se clasificaron a partir de cuatro variables. En primer lugar, actor interventor,

\footnotetext{
${ }^{1}$ Sistematización de experiencias de intervención social en el Municipio de Buenaventura y el Pacífico colombiano. Una línea de base para orientar la intervención estatal, privada y de cooperación internacional, en una sociedad después de los acuerdos de La Habana (Ibarra, et al., 2018).
} 
haciendo referencia a las entidades, instituciones estatales u organizaciones que ejecutaron el proyecto. En segundo lugar, el sector al que fueron dirigidos, cultura, educación, infraestructura, vivienda, salud e inclusión. En tercer lugar, los objetivos del proyecto. Por último, el sujeto intervenido, dividido en individuos, grupos, comunidades o sectores.

Las experiencias de intervención encontradas en los documentos fueron estudiadas con base en tres aspectos claves: lo que se quiere intervenir (problemas, causas, consecuencias), cómo intervenir (estrategias y recursos que se movilizan) y lo que se pretende alcanzar por medio de la intervención (el deber ser legitimado traducido en metas u objetivos). Estos aspectos, son tomados de la definición de la teoría del cambio "explica cómo se entiende que las actividades produzcan una serie de cambios de resultados que contribuyen a lograr los impactos finales previstos" (Rogers, 2014, p.13).

\section{EI contexto de la intervención: la región Pacífico y Buenaventura como objeto de intervención}

No es posible llegar a una comprensión íntegra de las intervenciones sin conocer las condiciones sociales, económicas y culturales en las cuales se desarrollaron. Se observa que, en el periodo 2000-2017, algunos tipos de intervención predominaron más que otros y que esto está ligado a hechos de contexto que influyeron en gran manera en la realidad social de Buenaventura. De acuerdo con esto, se identificaron principalmente tres subperiodos o momentos: el primero, entre el 2000 y 2005; el segundo, entre el 2006 y 2011; por último, el que va del 2012 al 2017. Cada uno marcado por algunas rupturas y continuidades.

El primer momento, se caracterizó por la creciente ola de violencia causada por la llegada masiva de paramilitares del Bloque Calima ${ }^{2}$ al municipio. Hecho que motivó al Estado a realizar acciones militares. Por otro lado, se impulsaba la construcción de nuevos puertos, tal como se registra en el Conpes 3342 Plan de Expansión portuaria del 2005. Contexto en el cual, la población civil bonaverense desarrolló acciones de resistencia, abocadas a la oposición, a la barbarie, defensa del territorio y los derechos humanos (CNMH, 2015).

En el año siguiente, con la elaboración del Conpes 3410 de 2006, el Estado se propuso priorizar a Buenaventura, ya no desde su pertenencia a la región Pacífica, sino desde lo municipal. Esta perspectiva, se ratifica con el nombramiento del municipio en Distrito Especial Portuario, Ecoturístico y Biodiverso en el año 2007. Entre este año y el 2011, al igual que el periodo anterior, se llevaron a cabo un gran número de acciones militares como respuesta al aumento de la violencia, especialmente en la zona urbana. A su vez, las organizaciones comunitarias desarrollaron iniciativas de paz y reconciliación, tal como la Capilla de la memoria, fundada en el 2009, y la marcha de jóvenes en contra la violencia del 2011.

\footnotetext{
${ }^{2}$ Entre los actos violentos de estos grupos armados ilegales se encuentran en las masacres del Naya, Citronela, Zaragosa, Yuramanguí, Anchicayá, Dagua, Sabaletas y los indígenas del Nasa. Además, de los desplazamientos masivos de la población rural (CNMH, 2015).
} 
En el 2012, con el inicio de los Diálogos de paz entre el Gobierno colombiano y las FARC-EP, se marcó un punto de ruptura, pues los temas de la paz, perdón y reconciliación se hicieron cada vez más recurrentes en las acciones de intervención, no solo de las organizaciones comunitarias, sino también del Estado. Como cierre de este periodo de tiempo, se resalta el Paro cívico desarrollado en el 2017, y que lleva por nombre vivir con dignidad y en paz en el territorio. En esta movilización, la población bonaverense se articuló con organizaciones, gremios y ONG operantes en el municipio, en señal de protesta por el continuo incumplimiento de los organismos gubernamentales.

\section{Descripción y análisis de resultados}

La construcción de la tipología, se realizó de manera descriptiva (Hillman, 2001), pues se abordó desde una realidad observable, a la cual se llegó principalmente a partir de fuentes documentales y casos representados en planes, programas, proyectos e iniciativas comunitarias. Dichas intervenciones, fueron analizadas principalmente a partir de los planteamientos teóricos de Corvalán (1996). Para este autor, la intervención social está dividida en cuatro tipos, caracterizados por la presencia diferenciada de las siguientes categorías. En primer lugar, la intervención nace de la interpretación de las necesidades humanas, las cuales pueden ser objetivables o subjetivas. Las primeras están definidas por el mercado y se encuentran estandarizadas por la teoría económica. Aquí, las necesidades de los receptores nacen de elementos estructurados fuera de ellos, es decir, de ideas universales ajenas a su realidad. Las necesidades subjetivas, por otro lado, son aquellas reconocidas por los mismos receptores, pues privilegian aspectos internos del individuo.

En segundo lugar, los distintos tipos de intervención pueden estar inspirados por una visión de la acción social colectiva o individual. La primera hace referencia a la movilización de recursos de un grupo de individuos, en torno a un conjunto reducido de objetivos definidos por el grupo y su logro es en consecuencia colectivo. La acción individual, en cambio, es la movilización de recursos psicológicos del individuo aislado. Esta acción, aun cuando puede ser común y simultánea con otros individuos, no obedece necesariamente a una concertación explícita de intereses individuales. Un claro ejemplo al respecto es el mercado, y a nivel de instituciones la empresa y la escuela.

En tercer lugar, de la interpretación del receptor (deficitarios o portadores). Los deficitarios son aquellos que protagonizan el problema que se intenta solucionar, presentan vacíos y tiene pocos elementos para cambiar su situación; los portadores, por su parte, poseen un conjunto de recursos de diferente índole para superar las problemáticas que los aquejan.

Por último, en cada tipo existe una priorización de un agente ideal o privilegiado de la intervención para formularla, orientarla y/o modificarla.

Tal como plantea Corvalán (1996), la forma en que se le da prioridad a unos u otros de los elementos ya mencionados, configura cuatro tipos de intervención: integrador, competitivo, militantista y movilizador (véase tabla 1). Cada uno de estos se sometió a un ejercicio analítico con las experiencias de intervención de Buenaventura. De esta manera, en los siguientes apartados se expone cada tipo con su respectiva discusión teórica y empírica. 
Tabla 1. Paradigmas sociales y sus concepciones en la intervención. Por Corvalán

\begin{tabular}{|c|c|c|c|c|}
\hline & Sujeto interventor & Sujeto intervenido & Estrategia & Perspectiva \\
\hline 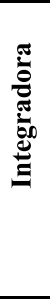 & $\begin{array}{l}\text { El Estado y sus } \\
\text { instituciones }\end{array}$ & $\begin{array}{l}\text { Se define a partir de } \\
\text { sus carencias, déficits. } \\
\text { Las principales } \\
\text { categorías usadas son: } \\
\text { marginales, desviados } \\
\text { y vulnerables. }\end{array}$ & $\begin{array}{l}\text { Política } \\
\text { pública }\end{array}$ & $\begin{array}{l}\text { Visión normativa y } \\
\text { objetivadora de la sociedad. } \\
\text { Esta intervención busca la } \\
\text { integración y } \\
\text { resocialización de los } \\
\text { "marginales" al proyecto } \\
\text { nacional. Parte del discurso } \\
\text { del acceso a la escolaridad } \\
\text { con los ingresos. }\end{array}$ \\
\hline 萢 & $\begin{array}{l}\text { Sector privado (la } \\
\text { empresa como } \\
\text { institución modelo) }\end{array}$ & $\begin{array}{l}\text { Definición a partir de } \\
\text { las posesiones. } \\
\text { Identifica a los } \\
\text { individuos como } \\
\text { estrategas, racionales y } \\
\text { capaces de elegir la } \\
\text { manera de satisfaces a } \\
\text { sus necesidades. }\end{array}$ & $\begin{array}{l}\text { Facilitar la } \\
\text { inserción al } \\
\text { mercado }\end{array}$ & $\begin{array}{l}\text { Presupone la necesidad de } \\
\text { potenciar la acción racional, } \\
\text { el uso de la libertad del } \\
\text { individuo y su acceso a las } \\
\text { instancias sociales de } \\
\text { intercambios simbólicos y } \\
\text { materiales. }\end{array}$ \\
\hline 营 & $\begin{array}{l}\text { Partido popular y } \\
\text { organizaciones políticas }\end{array}$ & $\begin{array}{l}\text { Define a los sujetos } \\
\text { como deficitarios, } \\
\text { busca potenciar la } \\
\text { acción del individuo o, } \\
\text { en otras palabras, que } \\
\text { tome conciencia de la } \\
\text { situación y se organice } \\
\text { para confrontar y } \\
\text { transformar el orden } \\
\text { capitalista. }\end{array}$ & $\begin{array}{l}\text { Acción } \\
\text { colectiva }\end{array}$ & $\begin{array}{l}\text { Privilegia la acción } \\
\text { colectiva sobre la } \\
\text { individual. El enfoque } \\
\text { colectivo a su vez no se } \\
\text { enfoca en una perspectiva } \\
\text { de inserción, sino de } \\
\text { confrontación. }\end{array}$ \\
\hline ن & $\begin{array}{l}\text { Organizaciones sociales, } \\
\text { sindicatos y } \\
\text { movimientos sociales }\end{array}$ & $\begin{array}{l}\text { Define a los sujetos } \\
\text { como portadores, } \\
\text { capaces de estructuras } \\
\text { un discurso de } \\
\text { reivindicación, de } \\
\text { organizar y producir } \\
\text { una lucha social, no } \\
\text { necesariamente para } \\
\text { cambiar la estructura } \\
\text { social. }\end{array}$ & $\begin{array}{l}\text { Proyectos } \\
\text { comunitarios y } \\
\text { programas } \\
\text { sociales. }\end{array}$ & $\begin{array}{l}\text { Intenta potenciar las } \\
\text { organizaciones y } \\
\text { movimientos sociales con el } \\
\text { objetivo de fortalecer su } \\
\text { capacidad de negociación o } \\
\text { de presión frente al poder } \\
\text { del Estado y otros grupos } \\
\text { sociales. También busca } \\
\text { que las organizaciones } \\
\text { logren convertirse en } \\
\text { movimientos sociales. }\end{array}$ \\
\hline
\end{tabular}

Fuente: elaboración propia con base en los planteamientos de Corvalán (1996).

\subsection{Intervención integradora}

De acuerdo con Corvalán (1996), la intervención integradora, es aquella en la que las necesidades son definidas como objetivables y homogéneas, con un acento en la integración a la vida social, democracia y acceso al conocimiento moderno, planteando el paso de la cultura tradicional a la moderna. En este tipo de intervención se le da gran valor al saber, siendo la difusión de la educación su principal herramienta. Así también, los procesos que lleva a cabo para cumplir su cometido son preferentemente de acción individual, apelando a categorías como ciudadanía y aprendizaje. Los receptores de la intervención o población intervenida se construyen a partir de sus carencias, como "deficitarios" (marginales, desviados, analfabetas, entre otros), los marginales o desviados son los actores para integrar. A nivel simbólico, se presupone la existencia de una cultura nacional, de modo que se conceptualiza al estado como agente central de 
integración y agente ideal para la intervención, como el único capaz de organizar la sociedad y resolver sus problemas.

Por lo que se refiere a la definición de este tipo y la intervención en Buenaventura, se identificó que algunas experiencias en sus objetivos contienen rasgos del tipo integrador, pero al mismo tiempo presentan características propias del contexto en el que se desarrolla. A continuación, esta particularidad se ilustra con algunas intervenciones propias de Buenaventura.

Para comenzar, los receptores de la intervención son construidos como deficitarios, pues se encuentran fuera de un todo debido a su condición de desigualdad social (Corvalán, 1996 y Carballeda, [2002] 2005). Así, por ejemplo, es común encontrar palabras como marginados o vulnerables, refiriéndose a los receptores de este tipo de intervención. Para ilustrar mejor, pensemos en el caso del estado colombiano, el cual, se ha centrado en la formulación de una serie de políticas sociales, orientadas a reducir o eliminar las condiciones de inequidad y marginación. Entre estas encontramos las políticas afirmativas que, atendiendo a estas condiciones, tienen como objetivo la inclusión y reconocimiento de diversos sujetos y colectivos. Buenaventura, al ser un municipio de población mayormente afrocolombiana, ha sido objeto de estas políticas (véase tabla 2).

Algunas de estas políticas afirmativas, se diseñaron y ejecutaron por el gobierno nacional con base en los artículos 1, 2, 7, 8, 10, 13, 17, 18, 19, 38, 40, 53, 63, 64, 65, 67, 70, $72,93,94,176,310,329,330$, AT.55 de la constitución política nacional de 1991. Dichos artículos parten del reconocimiento de la diversidad étnica y cultural del país. Esto, con el objetivo de reducir las brechas de desigualdad, por medio del mejoramiento de las condiciones de vida y oportunidades de desarrollo de la población afrocolombiana (Conpes $3310,2004)$.

Tabla 2. Política social para el Pacífico colombiano

\begin{tabular}{|l|l|}
\hline \multicolumn{1}{|c|}{ Conpes } & \multicolumn{1}{|c|}{ Objetivos } \\
\hline $\begin{array}{l}\text { 2902 de 1997: Programa de apoyo para el } \\
\text { desarrollo y reconocimiento étnico de las } \\
\text { comunidades Afro }\end{array}$ & $\begin{array}{l}\text { Apoyar el etnodesarrollo de las comunidades } \\
\text { afro. } \\
\text { Fortalecer las organizaciones de Comunidades } \\
\text { negras. } \\
\text { Defender los derechos étnicos, individuales y } \\
\text { colectivos de las comunidades afro. }\end{array}$ \\
\hline $\begin{array}{l}\text { 3169 de 2002: Política para la población } \\
\text { Afrocolombiana }\end{array}$ & $\begin{array}{l}\text { Mejorar las condiciones de vida de las } \\
\text { comunidades afrocolombianas y fortalecer los } \\
\text { procesos de titulación colectivas de tierras. } \\
\text { Completar y desarrollar los capítulos pertinentes } \\
\text { de la ley 70 de 1993. }\end{array}$ \\
\hline $\begin{array}{l}\text { 3310 de 2004: Política de acción afirmativa para la } \\
\text { población afrocolombiana }\end{array}$ & $\begin{array}{l}\text { Se somete a consideración una política de acción } \\
\text { afirmativa para la población afrocolombiana } \\
\text { orientada a focalizar acciones del Gobierno } \\
\text { nacional hacia estas comunidades. }\end{array}$ \\
\hline $\begin{array}{l}\text { 3660 del 2010: Política para promover la igualdad } \\
\text { de oportunidades afrocolombianas, palenquera y } \\
\text { raizal. }\end{array}$ & $\begin{array}{l}\text { Implementar soluciones para generar } \\
\text { oportunidades de acceso al desarrollo humano } \\
\text { sostenible y reducir la brecha en las condiciones } \\
\text { de vida de la población afrocolombiana. }\end{array}$ \\
\hline
\end{tabular}

Fuente: Elaboración propia a partir de revisión documental. 
Por otra parte, Corvalán (1996), expone que la educación es el medio principal para insertar a los individuos al proyecto nacional. Es decir, se insiste en el carácter unitario de la sociedad, por lo que la desviación, la heterogeneidad y la diversidad, aparecen como elementos pocos deseables. Para ello, se apelan a herramientas de aprendizaje, capacitación y formación con el fin de buscar la integración de los desviados a la sociedad. A diferencia de esto, el Estado colombiano exaltó la diversidad como parte del proyecto nacional a través del reconocimiento de país como un país pluriétnico y multicultural en la constitución de 1991. Por tanto, fue y sigue siendo imperativo ajustar las políticas o intervenciones a esta realidad social. Tal como se ha hecho desde algunos sectores con la etnoeducación, formalizada a través de la Ley 70 de 1993, del plan de desarrollo Hacia un Estado Comunitario.

De manera similar, pero bajo algunas particulares, se encuentran aquellos proyectos descritos en la Tabla 3, que enfatizan en la creación de un escenario de oportunidades para acceder a la educación, pero, al mismo tiempo, al mercado laboral. En Buenaventura estos se han desarrollado a partir de dos enfoques. Primero, el de formación artística para fortalecer la cultura del Pacífico. Segundo, el de fortalecimiento de habilidades con enfoque productivo (véase tabla 3). Este último punto evidencia que, en la especificidad de las intervenciones que se han realizado en el municipio, el tipo de paradigma de intervención social integracionista contiene rasgos del paradigma de intervención social competitivo, de acuerdo con la propuesta de Corvalan (1966), pues a través de la educación, se busca que los beneficiarios se incorporen en el mercado laboral y tengan mejores posibilidades de desarrollo productivo.

Tabla 3. Algunos proyectos del sector de la educación

\begin{tabular}{|c|c|c|}
\hline Proyectos & Actor & Entidades e instituciones \\
\hline Escuelas Lectoras (2014) & Privado & $\begin{array}{l}\text { Fundación Gases de Occidente y Sociedad } \\
\text { Portuaria }\end{array}$ \\
\hline $\begin{array}{l}\text { Programa de Incentivos a la } \\
\text { Calidad Educativa (2016) }\end{array}$ & Estado & Ministerio de Educación \\
\hline $\begin{array}{l}\text { Campaña Cuento Contigo } \\
(2013-2016)\end{array}$ & Estado y Privado & $\begin{array}{l}\text { Ministerio de Educación Nacional, Fundalectura } \\
\text { y Banco BBVA }\end{array}$ \\
\hline Escuelas Taller (2012) & $\begin{array}{l}\text { Estado, tercer } \\
\text { sector y Privado }\end{array}$ & $\begin{array}{l}\text { Ministerio de Cultura, SENA, Alcaldía distrital } \\
\text { de Buenaventura, AECID, USAID, OIM, CAF, } \\
\text { GIZ, Fundación Sociedad Portuaria de } \\
\text { Buenaventura, Fundación Carvajal, Fundación } \\
\text { Escuela Taller de Bogotá y diversas } \\
\text { organizaciones sociales locales y Ecopetrol. }\end{array}$ \\
\hline $\begin{array}{l}\text { En Buenaventura, los } \\
\text { jóvenes están "online" } \\
(2001)\end{array}$ & $\begin{array}{l}\text { Tercer sector y } \\
\text { Privado }\end{array}$ & $\begin{array}{l}\text { Fundación Sociedad Portuaria en alianza con la } \\
\text { universidad Eafit y Fundación People on line }\end{array}$ \\
\hline $\begin{array}{l}\text { Fomento a la } \\
\text { internacionalización de la } \\
\text { educación superior (2012) }\end{array}$ & $\begin{array}{l}\text { Estado y tercer } \\
\text { Sector }\end{array}$ & $\begin{array}{l}\text { Ministerio de Educación, Univalle sede Pacífico } \\
\text { (en Buenaventura) }\end{array}$ \\
\hline $\begin{array}{l}\text { Educación para la paz en el } \\
\text { Pacífico Colombiano (2015- } \\
\text { 2017) }\end{array}$ & Tercer Sector & $\begin{array}{l}\text { Agencia Española de la Cooperación } \\
\text { Internacional para el Desarrollo, Banco BBVA, } \\
\text { Universidad ICESI y Manos visibles }\end{array}$ \\
\hline $\begin{array}{l}\text { Biblioteca comunitaria } \\
(2017)\end{array}$ & Tercer Sector & Fundescodes \\
\hline
\end{tabular}

Fuente: Elaboración del grupo de investigación del proyecto general. 
En cuanto a la definición de necesidades dada por Corvalán(1996), se observa que estas se satisfacen por medio de bienes de consumo materiales, ayudas financieras o cualquier tipo de recurso que pretendan aportar una respuesta intermedia o final a varias necesidades. En el caso de la población bonaverense, estas se definen en relación con sus condiciones de pobreza, miseria y exclusión social. En consecuencia, las políticas sociales y económicas dirigidas a esta población, se han enfocado en suplir principalmente la falta de los servicios públicos, (agua potable, energía, alcantarillado, relleno sanitario y vivienda). Concretamente, en la construcción o mejoramiento de infraestructura social, con la partición del Estado y apoyo de agentes privados (véase tabla 4).

Ahora bien, aunque Corvalán no considera la acción militar como un rasgo del tipo integrador, se puede decir que en Buenaventura se ha constituido como un medio para insertar a los individuos al proyecto nacional. Así que, ante el fragmentado tejido social que ha dejado la violencia perpetrada por los grupos armados ilegales en Buenaventura, el Estado ha priorizado en acciones militares como medio principal para devolver el orden social. Algunas de estas intervenciones de tipo militar, corresponden a las políticas implementadas en el Gobierno de Álvaro Uribe en el Plan Hacia un Estado Comunitario entre 2002 y 2006, cuyo objetivo fue combatir a los grupos al margen de la ley, las bandas dedicadas al narcotráfico y la minería ilegal. Por otra parte, desde la administración local, las acciones se han concentrado en la vigilancia y control de actividades delictivas. En síntesis, esta forma de intromisión se relaciona con la definición de Weber (1979), del Estado como aquella institución humana que, dentro de un territorio reclama para sí el monopolio de la violencia física legítima. Al mismo tiempo, este aspecto de intervención por parte del Estado, corresponde a intereses privados relacionados con la disputa del control del territorio, referente a la construcción y expansión portuaria.

Tabla 4. Políticas, planes y proyectos para el NIB de la región

\begin{tabular}{|l|l|}
\hline \multicolumn{1}{|c|}{ Políticas, planes y proyectos } & \multicolumn{1}{c|}{ Énfasis de las intervenciones } \\
\hline Plan Pacífico (1994) & $\begin{array}{l}\text { Proyectos de infraestructura social (agua potable, energía, } \\
\text { saneamiento básico, educación, salud), desarrollo institucional } \\
\text { y actividades productivas. }\end{array}$ \\
\hline $\begin{array}{l}\text { Plan territorial del distrito de } \\
\text { Buenaventura }\end{array}$ & $\begin{array}{l}\text { Mejoramiento de la infraestructura del Hospital Distrital y de } \\
\text { colegios Atanasio Girardot y José Ramón Bejarano, } \\
\text { construcción de complejos deportivos. Adecuación de vías } \\
\text { para la movilidad. }\end{array}$ \\
\hline $\begin{array}{l}\text { Megaproyectos en infraestructura } \\
\text { educativa. }\end{array}$ & $\begin{array}{l}\text { Construcción del colegio San Antonio (Mega colegio), la } \\
\text { Ciudadela universitaria del Pacífico y la sede Náutica Pesquera } \\
\text { del Sena. }\end{array}$ \\
\hline Plan todos Somos Pacífico (2015) & Proporcionar energía eléctrica a zonas veredales. \\
\hline $\begin{array}{l}\text { 3410 de 2006: Política del Estado para } \\
\text { mejorar las condiciones de vida de la } \\
\text { población de Buenaventura }\end{array}$ & $\begin{array}{l}\text { Alcanzar los Objetivos del Milenio, promover el desarrollo } \\
\text { económico y la competitividad territorial. Convertir a } \\
\text { Buenaventura en una ciudad amable. }\end{array}$ \\
\hline $\begin{array}{l}3476 \text { de 2007: Importancia estratégica } \\
\text { de los macroproyectos de vivienda de } \\
\text { interés social en Cali y Buenaventura. }\end{array}$ & $\begin{array}{l}\text { El Macroproyecto de Vivienda de Interés Social, plantea la } \\
\text { reubicación de aproximadamente 3.400 hogares ya censados } \\
\text { que se encuentran localizados en zonas de alto riesgo en el } \\
\text { sector sur de la Isla Cascajal }{ }^{3} .\end{array}$ \\
\hline
\end{tabular}

Fuente: Elaboración propia a partir de la revisión documental.

\footnotetext{
${ }^{3}$ Este megaproyecto es cuestionable por los verdaderos intereses económicos de empresas portuarias con el reasentamiento de los pobladores en los territorios ganados al mar.
} 
En síntesis, los objetivos de la intervención en Buenaventura se han concentrado en la disminución de las brechas sociales y mejoramiento de las condiciones de vida de los bonaverenses, a través de políticas sociales y económicas, programas, proyectos e intervención militar. Sin embargo, como lo señalan las denuncias de sus pobladores e informes defensoriales, estas políticas no han tenido los resultados previstos. Esto se sustenta en que las condiciones de pobreza, exclusión social y desprotección han cambiado muy poco y en muchos casos se han agravado.

Por último, aunque el Estado colombiano ha sido el principal encargado de definir lo que se quiere intervenir, el contexto internacional ha jugado un papel importante en ello. Tal es el caso de los Objetivos del Milenio, ocho metas cuantificadas y cronológicas dirigidas a superar la pobreza extrema en sus distintas dimensiones, a partir de las cuales el gobierno ajustó sus planes de desarrollo en el marco de tiempo de duración de este acuerdo transnacional.

\subsection{Tipo de intervención competitiva}

Tal como afirma Corvalán (1996), este tipo de intervención busca que el mercado sea un mecanismo de inserción de los individuos al intercambio de bienes simbólicos y sociales. En Buenaventura, esta inserción se ha priorizado a nivel colectivo, desde una visión global del municipio en zona clave para el desarrollo económico del país. De este modo, se han realizado megaproyectos portuarios ${ }^{4}$ bajo alianzas entre el Estado y agentes privados, cuya contribución recae en guiar el municipio hacia al progreso y desarrollo. Una intervención que evidencia la modalidad de alianzas públicas-privadas en las que, según Martínez (2018), los agentes estatales crean corporaciones -empresas- de capital público privado. De ahí que, en ocasiones, sea difícil trazar una línea entre los recursos privados y públicos invertidos en estos megaproyectos.

Algo frecuente en los diferentes diagnósticos de los Conpes que se han formulado para la región Pacífica, es la definición de Buenaventura como principal puerto de carga del país, o en la única zona especial económica de exportación sobre el océano pacífico colombiano. Motivo por el cual la explotación de este potencial económico ha sido una constante en los discursos de las intervenciones realizadas a nivel institucional, atravesando incluso las visiones de transformación social.

Tal como se mencionó anteriormente, la política social es la principal estrategia del tipo integrador para lograr sus objetivos (Corvalán, 1996). Postura que parece diferir a la del tipo competitivo, cuyo principal medio de cambio es el mercado, pues existe una relación antagónica entre el Estado y el mercado, especialmente cuando se habla de la intromisión del primero en los asuntos macroeconómicos. De acuerdo con Teresa Montagut (2014), este es un enfoque conservador que plantea "la política social como una intromisión del Estado que altera y distorsiona las condiciones del mercado, causando inflación y desequilibrio" (p.22), razón principal por la que la intervención competitiva, en teoría, guardaría total independencia de la formulación de políticas sociales por parte del Estado.

${ }^{4}$ Entre estos se encuentran: Terminal de Contenedores de Buenaventura, Puerto Industrial Agua Dulce y la construcción de otros tres puertos nuevos el Puerto Delta del Río, Puerto Solo, Dagua. 
En Buenaventura, en cambio, varias de estas políticas privilegian la ejecución de megaproyectos y, consecuentemente, al capital privado. Basta como muestra la puesta en marcha de acciones como el dragado del canal de acceso a la bahía, la construcción de los puertos Aguadulce y TCBuen. Megaproyectos de infraestructura portuaria, que reflejan el juego de intereses existente por esta zona del país. Así pues, en este escenario, se ha gestado una disputa territorial entre los responsables de estos megaproyectos y la población que se encuentra asentada en las zonas que necesitan para construir. Un caso puntual, es el del megaproyecto Malecón Bahía de la Cruz. En este, los empresarios solo vieron esa iniciativa como una medida adecuada para promover el desarrollo de la ciudad; en cambio, la población que habitaba y trabajaba en la zona de construcción, estaba preocupada fundamentalmente porque se quedarían sin dónde vivir, sin el mar y, en consecuencia, sin fuentes de ingreso. En esta situación, además, hubo un desconocimiento por parte del Estado que esta zona del sur de la Isla Cascajal, fue ampliada por las comunidades afrodescendientes, las cuales rellenaron los espacios de marea baja hasta prologar la plataforma o hacer tierra, proceso que es conocido por sus pobladores como recuperación de territorios ganados al mar" (CNMH, 2015).

Ahora cabe señalar que, este tipo de intervención se expresa de otras formas, además de las ya mencionadas. En relación directa con el individuo, se busca que este potencie sus habilidades, para que le sea posible insertarse en el mercado y compitan en él con otros individuos. Bajo esta idea, se encuentran proyectos con enfoque productivo, los cuales se dirigen al fortalecimiento de actividades pesqueras, agropecuarias. Su principal perspectiva de cambio, es que los individuos sean agentes de su propio sustento, el cual les permite aproximarse a esta instancia de intercambio de bienes y servicios. Estos proyectos han sido ejecutados por el Estado, entidades privadas y organismos internacionales, los cuales también trabajan mediante alianzas (ver tabla 5).

Tabla 5. Algunos proyectos del sector productivo

\begin{tabular}{|l|l|l|}
\hline \multicolumn{1}{|c|}{ Proyecto } & \multicolumn{1}{|c|}{ Actor } & \multicolumn{1}{c|}{ Entidades e Instituciones } \\
\hline $\begin{array}{l}\text { Proyecto de } \\
\text { empleabilidad (2016) }\end{array}$ & Privado & Fundación Carvajal y Comfenalco \\
\hline $\begin{array}{l}\text { Fortalecimiento unidades } \\
\text { productivas (2016) }\end{array}$ & Privado & $\begin{array}{l}\text { Fundación Carvajal y Fundación Saldarriaga } \\
\text { Concha }\end{array}$ \\
\hline $\begin{array}{l}\text { SCOPE: Empleos para } \\
\text { Construir Futuro (2016) }\end{array}$ & $\begin{array}{l}\text { Estado, privado } \\
\text { y Tercer sector }\end{array}$ & $\begin{array}{l}\text { Fundación Carvajal, DPS, Gobierno de Canadá y } \\
\text { Cuso International }\end{array}$ \\
\hline $\begin{array}{l}\text { Pacífico Joven (2011- } \\
\text { 2015) }\end{array}$ & $\begin{array}{l}\text { Privado y tercer } \\
\text { sector }\end{array}$ & $\begin{array}{l}\text { Fundación Gases de Occidente, Fundación Surtigas } \\
\text { y SWISSAID }\end{array}$ \\
\hline $\begin{array}{l}\text { Proyecto Alianza de } \\
\text { pesca: Fortalecimiento } \\
\text { comercial y productivo de } \\
\text { la EAT la Bolichera }()\end{array}$ & $\begin{array}{l}\text { Estado, Privado } \\
\text { yercer sector }\end{array}$ & $\begin{array}{l}\text { Ministerio de Agricultura, Gobernación del Valle } \\
\text { SENA, Fundación Carvajal, Comercializadora }\end{array}$ \\
\hline $\begin{array}{l}\text { Programa de } \\
\text { empleabilidad (2013) }\end{array}$ & $\begin{array}{l}\text { Privado y Tercer } \\
\text { sector }\end{array}$ & $\begin{array}{l}\text { Fundación Carvajal, Fundación Coficolombiana, } \\
\text { Fundación Bancolombia, Comfenalco, Comfandi, } \\
\text { SENA, Cruz Roja, Actec Fundación Limmat y } \\
\text { ACDI VOCA }\end{array}$ \\
\hline $\begin{array}{l}\text { Mejorar las condiciones } \\
\text { de vida social y laboral de } \\
\text { las mujeres en situación } \\
\text { de vulnerabilidad de } \\
\text { Buenaventura }\end{array}$ & $\begin{array}{l}\text { Privado y Tercer } \\
\text { sector }\end{array}$ & $\begin{array}{l}\text { Fundación Mainel, Fundación Carvajal, Junta de } \\
\text { Castilla y León }\end{array}$ \\
\hline
\end{tabular}

Fuente: Elaboración del grupo de investigación del proyecto general. 
La idea de Estado que se atribuye a estos proyectos de intervención, se basa en este tipo de relación con el mercado para alcanzar sus objetivos individualistas al otorgar, como lo plantea la política neoliberal, libertad a los individuos para que alcancen de manera autónoma las capacidades que les permitan superarse frente a las dificultades (Montagut,2014). Así también, por medio de este mecanismo se ha buscado consolidar un Estado mínimo, para garantizar derechos sociales y máximo para el tránsito libre de capitales, generando nuevas interacciones entre lo público y privado.

Por último, es importante agregar que la responsabilidad social empresarial ha sido una de las principales herramientas, a través de la cual el sector privado ha intervenido en Buenaventura. Estos se han constituido en agremiaciones, por ejemplo, la Sociedad Portuaria Regional de Buenaventura y su respectiva fundación. De acuerdo con Benítez (2012), las empresas participan cada vez más en escenarios sociales y construyen nuevas agendas. Esto se puede visualizar, en los diferentes agentes privados que tienen presencia en Buenaventura, tales como la fundación Carvajal, Malecón y Fundación Agua Dulce, los cuales han tenido cierta incidencia en la orientación de cómo se debe intervenir en el municipio.

5.3. Intervención movilizadora con rasgos de tipo militantista: procesos colectivos y necesidades subjetivas

Para la construcción de este tipo de intervención, se retoman algunos rasgos de los tipos militantista y movilizador con sus respectivos paradigmas alineación y conflicto, descritos en la tabla 1. Concretamente, profundizando en las dimensiones de acción colectiva, necesidades subjetivas y la emergencia de organizaciones sociales. Es importante agregar que, si bien el paradigma de la alineación y su concepción de intervención militantista, se relacionan fuertemente con los postulados del marxismo, estos elementos no son aplicables para el caso de Buenaventura. Así pues, las organizaciones de las comunidades afro, más allá de cambiar el orden estructural de la sociedad, han dirigido sus acciones a su reconocimiento y legitimación estatal. Sobre esto habla Castillo (2017), quien se sitúa en la proclamación de la Constitución de 1991, para enfatizar el proceso de etnización de lo negro, con el apoyo directa e indirectamente de académicos, profesionales y líderes que participaron en la constituyente.

En síntesis, este tipo de intervención recoge los procesos colectivos de las organizaciones de base, los consejos comunitarios y cabildos indígenas que actúan en su territorio y se encargan de construir prácticas propias de transformación. Prácticas que, dentro del análisis de Corvalán (1996), se pueden llegar a denominar una forma de intervención. Estos se constituyen en proyectos sociales, que han emergido desde la base social, con el objetivo de ajustar las acciones externas a su realidad mediante alianzas entre diversos agentes interventores. En la tabla 6 se presentan algunos ejemplos. 
Tabla 6. Experiencias de intervención desde tres organizaciones

\begin{tabular}{|l|l|l|l|}
\hline Organizaciones & \multicolumn{1}{|c|}{ Campo en que actúan } & \multicolumn{1}{|c|}{ Estrategias } & Proyectos e iniciativas \\
\hline \multirow{2}{*}{ Anmucic } & $\begin{array}{l}\text { Defensa y protección } \\
\text { de la mujer en situación } \\
\text { de vulnerabilidad }\end{array}$ & $\begin{array}{l}\text { Recuperación y } \\
\text { fortalecimiento de los } \\
\text { lazos sociales. }\end{array}$ & Talleres de comadreo \\
\cline { 3 - 4 } Rostros Urbanos & $\begin{array}{l}\text { Creación de redes de } \\
\text { apoyo. }\end{array}$ & $\begin{array}{l}\text { Red de alimentos, } \\
\text { proyectos agrarios: } \\
\text { siembra en azotea de } \\
\text { plantas medicinales. }\end{array}$ \\
\hline \multirow{2}{*}{$\begin{array}{l}\text { Espacio } \\
\text { Humanitario }\end{array}$} & $\begin{array}{l}\text { Formación de jóvenes } \\
\text { participación y } \\
\text { liderazgo }\end{array}$ & $\begin{array}{l}\text { Arte y cultura (canto, } \\
\text { poesía, pintura y teatro) }\end{array}$ & $\begin{array}{l}\text { Talleres: Súmate por la } \\
\text { Paz, Recuperando la } \\
\text { Esquina por la Paz }\end{array}$ \\
\hline $\begin{array}{l}\text { Protección de los } \\
\text { la porechos Humanos de } \\
\text { Nayero la Playita }\end{array}$ & $\begin{array}{l}\text { Creación de un espacio } \\
\text { libre de actores } \\
\text { armados ilegales en la } \\
\text { zona urbana }\end{array}$ & $\begin{array}{l}\text { Esponformación del } \\
\text { el año 2014 }\end{array}$ \\
\hline
\end{tabular}

Fuente: presente investigación.

En estos tres casos, se destacan los enfoques diferenciados desde las perspectivas étnico-racial y cultural. Esto se materializa en acciones colectivas de protesta, a través de novedosos repertorios culturales y un tipo particular de gestión asociada en la que se articulan diversas organizaciones, asociaciones, sectores y población civil, en torno a distintas problemáticas que han afectado a la población, tal como la corrupción y la debilidad estatal en la zona, en asuntos referentes a la atención social integral, pero fuerte en materia militar y macroeconómica.

\subsection{Gestión Asociada}

Llegado este punto, es necesario tomar distancia de algunos planteamientos de Corvalán y, traer a la discusión otros, para referir a un tipo diferente de intervención. De manera puntual, la gestión asociada realiza una intervención que comprende la asociación entre actores sociales desde una perspectiva de iguales (sin imposiciones de poder) a partir de las diferencias de cada uno. Ruiz (2004), resalta que la gestión asociada, es una herramienta que permite lograr mayor equidad para los más desprotegidos, pues brinda un panorama en el que se promueve la creación de espacios de concertación en los que, en compañía de diversos actores (sector público, privado, no gubernamental y grupos de base), se construyen diagnósticos que permiten resolver problemas previamente detectados y priorizados por ellos. De este modo, la intervención intersectorial y multiactoral es positiva pues propende por el desarrollo conjunto de iniciativas.

Una experiencia de intervención con estas características es el proyecto: fortaleciendo a la sociedad civil para la Gobernanza "transformando relaciones para la paz". Tiene como objetivo, consolidar la paz en los territorios y así restaurar y transformar estas relaciones disfuncionales, entre las estructuras del Estado y la sociedad civil. Para lograrlo, el Programa propone llegar a un grupo focal de ciento once organizaciones de sociedad civil, incluyendo diecisiete Organizaciones de Apoyo Intermedio (OAI), estas son las aliadas Pastorales Sociales en las regiones priorizadas, y noventa y cuatro organizaciones de los municipios focalizados. 


\section{Discusión conceptual y valoración de hallazgos}

Llegado a este punto, el ejercicio analítico propuesto entre los tipos de Corvalán (1996), y las experiencias de intervención en Buenaventura, lleva a replantear sus definiciones, con el objetivo de captar particularidades propias del contexto en que se desarrollan. De esta manera surgieron dos importantes que no se contemplan en la tipología, que a continuación se describirán.

En primer lugar, cada tipo no es representado únicamente por un agente interventor y no siempre es el actor ideal que propone Corvalán (1996). Se identifican diferentes actores involucrados en un mismo tipo; por ejemplo, los proyectos económicos se llevan a cabo no solo por instituciones privadas, sino también por instituciones estatales con los que establecen alianzas. En referencia a esto, tanto el Estado como el sector empresarial han impulsado megaproyectos portuarios. Así mismo, el Estado lleva a cabo intervenciones mixtas con otros actores, quienes se involucran en estos proyectos como inversionistas u operadores.

En segundo lugar, respecto al sujeto intervenido para Buenaventura no solo es deficitario, sino que juega un doble rol, también es portador. Aquel individuo con la capacidad de superar dificultades por sí mismo. Como muestra, se encuentran proyectos desarrollados por medio del Sena o el programa de transferencias Jóvenes en acción. En el primero, utiliza como principal herramienta la educación, pero que apunta a lo productivo, a que los beneficiarios aprendan una actividad u oficio para que construyan emprendimientos. En el segundo, brinda apoyo financiero a las personas vulnerables, con el propósito de que fortalezcan sus capacidades y logren superarse por sí mismos.

\section{Conclusiones}

Lo dicho hasta aquí, revela que la ejecución de intervenciones no siempre parte de una definición de las necesidades reales de la población, sino que se basa en modelos foráneos preestablecidos. De este modo, la concepción de intervención que se ha dado en Buenaventura parte de un contexto regional, donde la doctrina de desarrollo ha regido gran parte de las políticas ejecutadas. Lo que tiene su raíz en la "construcción discursiva de los problemas sociales y el tipo de soluciones políticas que se implementan para resolver dichos problemas" (Amaya y Portilla, 2016, p.39).

Ahora, es necesario dar un repaso por la noción de desarrollo, que suele estar ligada a las intervenciones que llevan a cabo en Buenaventura. Escobar y Pedrosa (1996), reconocen el desarrollo como paradigma y práctica. Para estos autores, el discurso del desarrollo tiene la característica de colonizar las mentes de los dirigentes del país, quienes insisten en prácticas y esquemas que se quiere aplicar al Pacífico colombiano. Todo esto se expresa en programas, estrategias de desarrollo económico, rural, urbano, o en las áreas de salud, nutrición, y educación; conocimientos expertos y especializados; al igual que en relaciones con entidades internacionales como el Banco Mundial.

De ahí que, el cumplimiento de sus objetivos no siempre resulte en un mejoramiento de las condiciones de vida de los sujetos intervenidos. Debido a esto, es imperativo un trabajo articulado, como el que se propone desde el planteamiento de la gestión asociada, en el cual los distintos actores nutren las intervenciones comunitarias a través de la 
unión de esfuerzos. En el desarrollo de este tipo de iniciativas, se rompe la dicotomía entre interventor-intervenido y se desarrollan procesos basados en modelos híbridos que combinan varias perspectivas, estrategias y actores.

Conviene subrayar que, a partir del estudio de estos tipos de intervención, fue posible evidenciar que el modelo predominante de intervención en Buenaventura, es aquel que posee rasgos del tipo integrador con un enfoque competitivo. Este tipo corresponde a una perspectiva de intervención social, en la cual no hay solución de continuidad clara entre el propósito de integrar a la población a un todo y el de aprovechamiento económico del municipio, a partir de sus riquezas naturales y el puerto; por tanto, planteamientos teóricos como el de Corvalán (1996), tienen el fin de integrar a las personas a la sociedad, por ejemplo, a través de la educación. En este nuevo enfoque la educación tendría además un propósito diferente: el de formar personas con ciertas capacidades, que fortalezcan su proceso de individuación dentro de la sociedad.

Los cuatro tipos propuestos para Buenaventura, comprenden una disputa entre las formas de pensar e incidir en la transformación social. La cual ha sido diferenciada, es decir, desde actores como el Estado y el sector empresarial, se han dado modos muy específicos de plantear la intervención. Dichos modos, se constituyen en metodologías de intervención que buscan legitimar por medio de su posición de poder. En este sentido, han desplegado una serie de políticas y planes a nivel nacional y regional, que adoptan una visión hegemónica de desarrollo económico, como eje central de sus intervenciones. Esta lógica, contrasta con la de las organizaciones sociales o consejos comunitarios locales, pues estos actores se apoyan en sus tradiciones y visión ancestral del mundo que los rodea y, por tanto, parten de una definición subjetiva de sus necesidades. Así, tanto el Estado, como los agentes privados y las organizaciones sociales, poseen distintas visiones de cambio y recursos que les otorgan ventajas y desventajas para alcanzar sus objetivos en el contexto de Buenaventura.

\section{Referencias Bibliográficas}

Amaya, J., y Portilla, R., (2016). Marcos interpretativos de políticas públicas. El enfoque preventivo de la seguridad en el discurso político de los candidatos a presidentes municipales de Guadalajara, Zapopan, Jalisco México, en las elecciones de 2015. Collectivus, Revista de Ciencias Sociales, 3(1), 36-65. DOI: https://doi.org/ 10.15648/coll.1.2016.3

Carballeda, A. [2002] (2005). La intervención en lo social; exclusión e integración en los nuevos escenarios sociales. Buenos Aires, Argentina: Editorial Paidós SAICEF y Tataki S.A.

Castillo, L. (2006). El Estado-Nación pluriétnico y multicultural colombiano: La lucha por el territorio en la imaginación de la Nación y la reivindicación de la identidad étnica de negros e indigenas (tesis doctoral). Universidad Complutense de Madrid, España.

Centro Nacional de Memoria Histórica. (2015). Buenaventura: un puerto sin comunidad. Bogotá D.C., Colombia: Autor. 
Contralora Distrital de Buenaventura (2017). Informe anual del estado de los recursos naturales y medio ambiente en Buenaventura.

Corvalán, J. (1996). Los paradigmas de lo social y las concepciones de intervención en la sociedad (Documento $\mathrm{N}^{\circ} 4$, CIDE).

DANE (2018). Medida de pobreza multidimensional municipal de fuente censal 2018. Recuperado de https://www.dane.gov.co/index.php/estadisticas-por-tema/pobreza-ycondiciones-de-vida/pobreza-y-desigualdad/medida-de-pobreza-multidimensional-defuente-censal

Defensoría del Pueblo (2016). Subregión Valle del Cauca-Buenaventura.

Escobar, A. (2004). Desplazamientos, desarrollo y modernidad en el Pacífico colombiano. Conflicto e (in) visibilidad. En E. Restrepo y A. Rojas (Eds.), Retos en los estudios de la gente negra en Colombia (p. 53-72). Popayán, Colombia: Editorial Universidad del Cauca.

Espinosa, A. (2011). De lo global a lo local en los repertorios de acción de las organizaciones negras frente al conflicto armado en Buenaventura. Universidad del Valle, facultad de ciencias sociales y económicas.

Hillman, K. (2001). Diccionario enciclopédico de sociología. Papers: revista de sociología, (núm. 48).

Martínez, A. (2018). Empresarialismo y grandes proyectos urbanos: análisis comparativo de puerto madero en buenos aires y la loma en Barranquilla. Collectivus, Revista de Ciencias Sociales, 5(2), 80-108. DOI: https://doi.org/10.15648/Coll.2.2018.6

Montagut, T. (2014). Que es política social En Política social: una introducción (pp. 1929), España, Barcelona: Grupo Planet (GBS).

Oslender, U. [1999] (2012). Espacializando la resistencia: perspectivas de espacio y lugar en las investigaciones de movimientos sociales. Revista Colombiana de Geografía, 8 (1), $1-35$.

Rogers, P. (2014). La teoría del cambio, Síntesis metodológicas: evaluación de impacto $\mathrm{N}^{\mathrm{o}}$ 2, Centro de Investigaciones de UNICEF, Florencia: Editor.

Ruiz, V. (2004). Organizaciones comunitarias y gestión asociada. Una estrategia para la ciudadanía emancipada. Buenos Aires, Argentina: Paidós.

Tancara Q, Constantino. (1993). La investigación documental. Temas Sociales No17. Recuperado de http://www.scielo.org.bo/scielo.php?script=sci_arttext\&pid=S004029151993000100008\&lng=es\&tlng=es. 